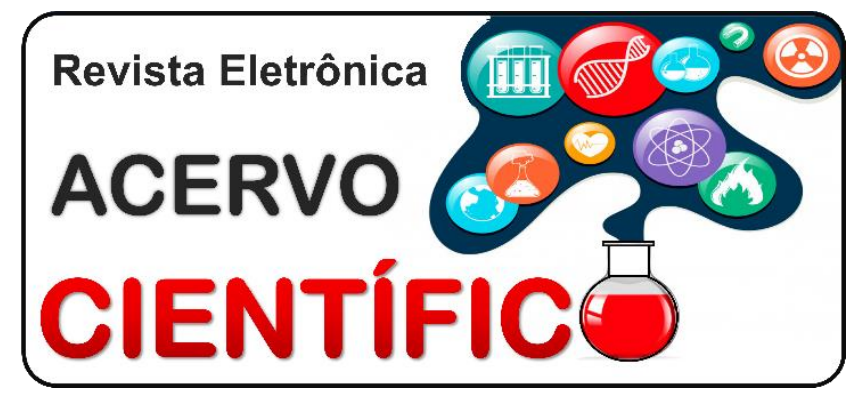

\section{REVISÃO BIBLIOGRÁFICA}

Recebido em: 4/2020

Aceito em: $5 / 2020$

Publicado em: $8 / 2020$

\title{
Vivências e percepções de mães de portadores de fibrose cística: uma perspectiva do cuidado
}

\author{
Experiences and perceptions of mothers with cystic fibrosis patients: a care perspective
Experiencias y percepciones de madres con pacientes con fibrosis cística: una perspectiva de cuidado

Adilson Mendes de Figueiredo Júnior ${ }^{1 *}$, Glaucilene Viana Santa Brigida1, Mateus Augusto do Amaral Castro ${ }^{1}$, Ingrid Inez dos Santos Amaral ${ }^{1}$, Bruna dos Santos da Silva ${ }^{1}$, Alan Daniel Reis de Sá ${ }^{1}$, Cristiane dos Santos Silva ${ }^{2}$, Ana Beatriz de Oliveira Vieira ${ }^{2}$, Barbara Alves Ruela de Azevedo Ruivo ${ }^{3}$, Ana Rosa Tavares Paixão ${ }^{3}$.

\begin{abstract}
Resumo: Esse artigo buscou identificar os sentimentos envolvidos de familiares durante a assistência em saúde de portadores de fibrose cística. A Fibrose Cística (FC) é uma doença genética descrita em 1930 como uma doença infantil com baixa expectativa de vida. Mesmo com avanços no diagnóstico e tratamento, a FC ainda apresenta inúmeras dificuldades associadas, que variam desde o acesso a serviços de saúde até as reações multisistêmicas. Tratou-se de um estudo de natureza qualitativo-descritiva sob método de revisão bibliográfica desenvolvida por meio do método da revisão integrativa da literatura. As mães de pacientes com FC vivenciam maior estresse e pior adaptação que as mães de crianças saudáveis ou que a população geral. Uma recente meta-análise demonstrou que as mães de crianças com doenças crônicas apresentavam uma prevalência de Transtorno de Estresse Pós-Traumático significativamente maior que os pais. As intervenções de enfermagem orientadas ao seguimento do adolescente ao longo do processo terapêutico necessitam priorizar os pais/família de forma significativa, considerando que o cuidado é holístico e fundamentado na subjetividade do indivíduo.
\end{abstract}

Palavras-chave: Enfermagem, Família, Fibrose cística.

\begin{abstract}
This article sought to identify the feelings involved in family members during health care for patients with cystic fibrosis. Cystic Fibrosis (CF) is a genetic disease described in 1930 as a childhood disease with low life expectancy. Despite advances in diagnosis and treatment, CF still has numerous associated difficulties, ranging from access to health services to multisystemic reactions. It was a qualitativedescriptive study under the bibliographic review method developed using the integrative literature review method. Mothers of CF patients experience greater stress and worse adaptation than mothers of healthy children or the general population. A recent meta-analysis showed that mothers of children with chronic illnesses had a significantly higher prevalence of Posttraumatic Stress Disorder than fathers. Nursing interventions aimed at following the adolescent throughout the therapeutic process need to prioritize parents / family significantly, considering that care is holistic and based on the subjectivity of the individual.
\end{abstract}

Keywords: Nursing, Family, Cystic fibrosis.

\footnotetext{
1 Escola Superior da Amazônia (ESAMAZ), Belém - Pará. *E-mail: adilsonmdfj@hotmail.com

2 Universidade do Estado do Pará (UEPA), Belém - Pará.

${ }_{3}^{3}$ Universidade Federal do Pará (UFPA), Belém - Pará.
} 
Resumen: Este artículo buscaba identificar los sentimientos involucrados en los miembros de la familia durante la atención médica para pacientes con fibrosis quística. La fibrosis quística (FQ) es una enfermedad genética descrita en 1930 como una enfermedad infantil con baja esperanza de vida. A pesar de los avances en el diagnóstico y el tratamiento, la FQ todavía tiene numerosas dificultades asociadas, que van desde el acceso a los servicios de salud hasta las reacciones multisistémicas. Fue un estudio cualitativodescriptivo bajo el método de revisión bibliográfica desarrollado utilizando el método integrador de revisión de literatura. Las madres de pacientes con FQ experimentan mayor estrés y peor adaptación que las madres de niños sanos o la población en general. Un metaanálisis reciente mostró que las madres de niños con enfermedades crónicas tenían una prevalencia significativamente mayor de trastorno de estrés postraumático que los padres. Las intervenciones de enfermería destinadas a seguir al adolescente a lo largo del proceso terapéutico deben priorizar significativamente a los padres / familia, considerando que la atención es holística y se basa en la subjetividad del individuo.

Palabras clave: Enfermería, Familia, Fibrosis quística.

\section{INTRODUÇÃO}

A Fibrose Cística (FC) é uma doença genética descrita em 1930 como uma doença infantil com baixa expectativa de vida. Mesmo com avanços no diagnóstico e tratamento, a FC ainda apresenta inúmeras dificuldades associadas, que variam desde o acesso a serviços de saúde até as reações multisistêmicas causadas pela mesma que englobam obstrução intestinal ou diarreia, tosse crônica com secreção, transpiração excessiva com a presença de suor hipertônico, problemas respiratórios e deformidade nos dedos e unhas (SILVA A, et al., 2015).

As mutações genéticas da fibrose cística levam às complicações diferentes, as principais são problemas nos pulmões e no sistema gastrointestinal, sendo a principal causa de mortalidade as complicações pulmonares. Inúmeros são os problemas relacionados à doença e, dessa forma, o tratamento se torna complexo e elaborado (REIS AT, et al., 2016).

A doença avança no segmento biológico, mas pouco ainda é visto quando consideramos o conceito completo de saúde que aborda o equilíbrio biológico, mental e social. A identificação e aconselhamento de familiares sobre esse transtorno recessivo comum é importante para detecção de casos adicionais e planejamento reprodutivo, além de permitir apoio e assistência não só ao paciente, mas aos familiares que estão inseridos no processo do cuidar que, muitas vezes, é caracterizado por um processo de esgotamento físico e mental (SCHRAM C, 2012).

A concepção de equilíbrio em saúde deve permutar os diferentes alicerces que sustentam o individuo entre os quais, a família continua sendo um dos mais importantes. O profissional da enfermagem por ser um educador social e praticante direto do cuidar deve aguçar sua percepção assistencial com a finalidade de englobar todos aqueles que direta e indiretamente se relacionam com o paciente (TAVARES KO, et al., 2010).

A família tem que ser vista como um complemento e é considerada uma parte imprescindível no cuidado do doente, e vivenciam a doença em todas as suas fases tentando se adaptar à nova condição de vida que vão enfrentar com o doente. Esse processo acontece de maneira gradual, pois não é uma tarefa fácil perceber a nova perspectiva de vida da criança restaurando sua dignidade (SILVA A, et al. 2013).

Por muito tempo, a família se manteve afastada dos processos de assistência em saúde e, de maneira imperceptível, os profissionais envolvidos no cuidado não avaliavam a necessidade de abordagem de todos que fazem parte do contexto saúde doença. A criação de protocolos de atendimento, além das ações de educação continuada e educação em saúde são meios que podem ajudar na melhor inserção dos familiares nos diversos setores de saúde (CABIZUCA M, et al., 2010).

O profissional de saúde que trabalha no ambiente hospitalar se encontra diretamente ligado às emoções dos familiares de seus pacientes em tratamento e de toda equipe, assumindo assim grande importância. Assim, o profissional enfermeiro que atua nessa área é responsável pela avaliação e prevenção de 
possíveis complicações no quadro clínico, assim como por intervenções de tratamento que podem variar do tratamento medicamento às vivências familiares (REIS AT, et al., 2016).

Muitos avanços já ocorreram nos estudos da fisiopatologia e etiopatogenia da fibrose cística, no Brasil, apenas $25 \%$ dos portadores da patologia possuem mais de 18 anos de idade. Pelas características sociais, biológicas e até mesmo mentais que permeiam o público em questão há uma necessidade de investigações que possam avaliar os espaços existentes na assistência de enfermagem ou multiprofissional. Dentre as necessidades em questão, pode-se citar a relação familiar e a interpretação dos desafios que os familiares enfrentam frente à doença (REIS AT, et al., 2016).

O cuidado ao paciente fibrocístico menor de idade não envolve apenas a doença, mas também o familiar que o acompanha, representado na maioria das vezes, pela mãe. Essa assume o principal papel de cuidadora e devido à cronicidade do agravo e as complexidades existentes, a mãe acaba vivendo diversas dificuldades, inclusive o sofrimento pelo diagnóstico, o número reduzido de locais especializados para o tratamento, preconceito das pessoas, frequentes hospitalizações, entre outras. Entender essas dificuldades é respeitar a complexidade do cuidado tornando-o pleno e eficaz (REIS AT, et al, 2016; CABIZUCA M, et al., 2010).

Muitas vezes, as equipes de saúde direcionam as abordagens para os fatores biológicos do indivíduo com a fibrose cística, o que gera um crescimento apenas técnico do aspecto do cuidar, e dessa forma, esquecem que as necessidades que formam o ser-humano são bem maiores que as questões físicas. É fundamental conhecer as peculiaridades, não só do paciente, mas da mãe para que o profissional da enfermagem apresente outro olhar em relação ao processo saúde-doença dos indivíduos que apresentam a fibrose cística e não apenas a visão biomédica (TAVARES KO, et al., 2010).

O objetivo do estudo foi identificar os sentimentos envolvidos de familiares durante a assistência em saúde de portadores de fibrose cística.

\section{MÉTODOS}

Tratou-se de um estudo de natureza qualitativo-descritiva sob método de revisão bibliográfica desenvolvida por meio do método da revisão integrativa da literatura, cujo objetivo foi proporcionar a incorporação das evidências de estudos a partir do tema "Sentimentos envolvidos de familiares durante a assistência em saúde de portadores de fibrose cística" para que se constitua um importante elo na promoção das interações, para busca das melhores estratégias e políticas que possibilite um cuidado holístico aos profissionais envolvidos. Os artigos foram agrupados e lidos atenciosamente para identificação do objetivo da pesquisa, após leitura os artigos foram selecionados e separados de acordo com sua natureza e objeto de estudo.

Como critérios de inclusão foram incluídos artigos completos que contemplem o tema e objetivo da pesquisa, que tenha relevância ao tema proposto do projeto da pesquisa dentre os anos de 2012 a 2018, produzidos no Brasil e em português. Como critérios de exclusão foram excluídos artigos que tenham como tema sentimentos envolvidos de familiares durante a assistência em saúde de portadores de fibrose cística com outras patologias associadas; artigos que necessitem que alguma taxa de pagamento para serem lidos e artigos que relacionem outros temas além de Fibrose Cística.

Os riscos da pesquisa foram mínimos, pois tratou-se de uma revisão bibliográfica, tais riscos foram representados por uma possibilidade de troca de informações e seus respectivos autores durante a discussão do projeto, para minimizar tais possibilidades todos os artigos científicos foram organizados em pastas e lidos individualmente para não haver conflito de idéias que pudessem confundir os pesquisadores.

Com os resultados do estudo, visa-se contribuir para incrementar os conhecimentos a respeito da assistência aos familiares dos portadores de Fibrose Cística. Espera-se também que ocorra melhoria das ações desenvolvidas pelos profissionais da área da saúde, mais especificamente os enfermeiros que atuam na área de atendimento à portadores de FC e seus familiares. 


\section{RESULTADOS}

Os dados coletados foram organizados em foram de tabelas (Quadro 1), onde forma tabuladas informações que envolveram o título do artigo, estado onde foram publicados, ano de publicação e objetivo geral de cada estudo.

Quadro 1 - Características dos artigos científicos que abordam fatores relacionados aos sentimentos envolvidos de familiares durante a assistência em saúde de portadores de fibrose cística.

\begin{tabular}{|c|c|c|c|}
\hline $\begin{array}{l}\text { Título do } \\
\text { Artigo }\end{array}$ & Estado & Ano & Objetivo geral \\
\hline $\begin{array}{l}\text { O que é ser mãe de } \\
\text { uma criança com fibrose } \\
\text { cística }\end{array}$ & $\begin{array}{l}\text { Rio Grande do } \\
\text { Sul }\end{array}$ & 2010 & $\begin{array}{c}\text { Conhecer a vivência de ser mãe de uma criança } \\
\text { com fibrose cística. }\end{array}$ \\
\hline $\begin{array}{l}\text { Vivências de familiares } \\
\text { de crianças e } \\
\text { adolescentes com } \\
\text { fibrose cística }\end{array}$ & Pernambuco & 2010 & $\begin{array}{l}\text { Conhecer e descrever as vivências de familiares de } \\
\text { crianças e adolescentes com fibrose cística. }\end{array}$ \\
\hline $\begin{array}{l}\text { Os pacientes invisíveis: } \\
\text { transtorno de estresse } \\
\text { pós-traumático em pais } \\
\text { de pacientes com } \\
\text { fibrose cística } \\
\end{array}$ & Rio de Janeiro & 2010 & $\begin{array}{c}\text { Verificar a prevalência de TEPT (Transtorno de } \\
\text { Estresse Pós Traumático) e dos três grupos de } \\
\text { sintomas de estresse pós-traumático em pais de } \\
\text { pacientes com fibrose cística. }\end{array}$ \\
\hline $\begin{array}{l}\text { Notícias difíceis: } \\
\text { sentidos atribuídos por } \\
\text { familiares de crianças } \\
\text { com fibrose cística }\end{array}$ & Rio de Janeiro & 2013 & $\begin{array}{l}\text { Apreender sentidos atribuídos por familiares de } \\
\text { crianças com fibrose cística sobre as notícias } \\
\text { difíceis transmitidas por profissionais de saúde } \\
\text { durante o tratamento num hospital público terciário } \\
\text { da zona sul do Rio de Janeiro. }\end{array}$ \\
\hline $\begin{array}{l}\text { Viver com fibrose } \\
\text { cística: a visão pessoal } \\
\text { do adolescente } \\
\text { brasileiro }\end{array}$ & Paraná & 2016 & $\begin{array}{l}\text { Examinar os desafios psicológicos de adolescentes } \\
\text { com fibrose cística (FC) no Brasil, por meio de uma } \\
\text { entrevista semiestruturada com perguntas abertas. }\end{array}$ \\
\hline $\begin{array}{l}\text { Vivências de mães que } \\
\text { acompanham filhos com } \\
\text { fibrose cística no } \\
\text { hospital: subsídios para } \\
\text { a enfermagem }\end{array}$ & São Paulo & 2016 & $\begin{array}{l}\text { Compreender as vivências de mães que } \\
\text { acompanham a hospitalização de seus filhos com } \\
\text { fibrose cística. }\end{array}$ \\
\hline $\begin{array}{l}\text { Intervenções de } \\
\text { enfermagem no } \\
\text { monitoramento de } \\
\text { adolescentes com } \\
\text { fibrose cística: uma } \\
\text { revisão da literatura }\end{array}$ & São Paulo & 2016 & $\begin{array}{c}\text { Buscar intervenções de enfermagem que enfoquem } \\
\text { a melhoria da qualidade de vida e a promoção do } \\
\text { autocuidado em adolescentes que sofrem de } \\
\text { fibrose cística. }\end{array}$ \\
\hline
\end{tabular}

Fonte: Júnior AMF, et al., 2018.

As produções que envolveram fatores relacionados aos sentimentos envolvidos de familiares durante a assistência em saúde de portadores de fibrose cística se concentraram principalmente nas regiões SulSudeste e em uma menor quantidade nas regiões Norte-Nordeste, distribuídos nos estados de São Paulo (28,7\%), no estado do Rio de Janeiro $(28,7 \%)$, no estado do Rio Grande do Sul $(14,2 \%)$, no estado do Paraná $(14,2 \%)$ e no estado de Pernambuco $(14,2 \%)$. Os achados científicos foram publicados nos anos de 2010 (42,6\%), ano de $2013(14,8)$ e ano de 2018 (42,6\%) de acordo com a Tabela 1. 
Tabela 1 - Distribuição dos artigos científicos que abordam os fatores relacionados aos sentimentos envolvidos de familiares durante a assistência em saúde de portadores de fibrose cística - Região de Publicação x Ano.

\begin{tabular}{lcccc}
\hline \multirow{2}{*}{ Ano de publicação } & \multicolumn{3}{c}{ Artigos científicos } \\
\cline { 2 - 5 } & \multicolumn{2}{c}{ Sul-Sudeste } & Norte-Nordeste \\
\cline { 2 - 5 } & Quantidade & $\%$ & Quantidade & $\%$ \\
\hline 2010 & 2 & 28,70 & 1 & 14,50 \\
2013 & 1 & 14,20 & 00 & 0 \\
2016 & 3 & 42,60 & 00 & 0 \\
\hline Total & 6 & 85,50 & 1 & 14,50 \\
\hline
\end{tabular}

Fonte: Júnior AMF, et al., 2018.

Observa-se que as produções que envolvem os fatores relacionados aos sentimentos envolvidos de familiares durante a assistência em saúde de portadores de fibrose cística foram produzidas em sua maioria nas regiões Sul-Sudeste, fato esse, que demonstra a deficiência dos estudos na região Norte. A maioria as produções encontradas foram publicadas nos últimos 5 anos, ressaltando a importância da atualização e periodicidade das investigações científicas que possam envolver o assunto em questão para que ocorra sempre um dinamismo na forma de observar e interpretar os fatores relacionados a saúde do doente e da família. Os sentimentos mais encontrados durante o estudo (Tabela 2) foram a angústia e/ou desespero com $71,40 \%$.

Tabela 2 - Sentimentos envolvidos de familiares durante a assistência em saúde de portadores de fibrose cística.

\begin{tabular}{lcc}
\hline \multirow{2}{*}{ Sentimentos } & \multicolumn{2}{c}{ Grupo de atenção } \\
\cline { 2 - 3 } & \multicolumn{2}{c}{ Família } \\
\cline { 2 - 3 } & Quantidade & $\%$ \\
\hline Angústia/Desespero & 5 & 71,40 \\
Medo da Morte & 1 & 14,30 \\
Imparcialidade & 1 & 14,30 \\
\hline Total & 7 & 100,00 \\
\hline
\end{tabular}

Fonte: Júnior AMF, et al., 2018.

\section{DISCUSSÃO}

Atualmente as novas abordagens da doença e o avanço da tecnologia aumentaram significativamente a expectativa de vida dos pacientes com Fibrose Cística (FC) que era de aproximadamente 15 anos especialmente nos países desenvolvidos, mas ela continua sendo considerada uma doença letal, sendo o agravo pulmonar a principal causa de morte. Suas manifestações costumam ocorrer desde os primeiros meses de vida, entretanto o diagnóstico, muitas vezes, não é imediato, no Brasil ainda ocorrem demoras no processo de investigação. Diagnosticada, a FC requer tratamento intensivo e uma rotina rigorosa de cuidados não só biológicos, mas sociais e mentais (AFONSO SBC e MITRE RMA, 2013).

Em grande maioria, muitos indivíduos experimentam doenças como um ataque à sua integridade física e mental. Além disso, esta situação vivida na infância e na adolescência pode desencadear uma crise de sobreposição de valores e até a perda da identidade social. Em outras palavras, soma-se à crise do corpo na doença e alterações psicológicas inerentes à própria fase de desenvolvimento da adolescência que, por muitas vezes, representa um leque de emoções. Os cuidados de maneira holística devem ser intensificados, pois a adolescência é o estágio de desenvolvimento psicológico que pode ser difícil e frustrante para o jovem saudável. Além disso, para os jovens com a doença, a adolescência e 0 desenvolvimento psicológico podem ser decepcionantes, levando a frustrações graves (AGUIAR KCA, et al., 2016).

Os familiares de forma geral, mas principalmente as mães de pacientes com FC, vivenciam maior estresse e pior adaptação que as mães de crianças saudáveis ou que a população geral. Inúmeros estudos 
atuais demonstram que as mães de crianças com doenças crônicas apresentavam uma prevalência de Transtorno de Estresse Pós-Traumático (TEPT) significativamente maior que os pais. As mães geralmente são mais afetadas que os pais, provavelmente porque elas são as cuidadoras primárias e estão mais envolvidas no tratamento desde o nascimento até a maturidade do ser humano (CABIZUCA M, et al., 2010).

De acordo com o estudo de Costa ASM, et al. (2010), a doença, para os familiares de crianças e adolescentes, se caracteriza como uma jornada longa e cheia de medos, principalmente, pelas incertezas da expectativa de vida e das possíveis complicações da doença. Quando o diagnóstico é concretizado, os sentimentos de desespero e dor se tornam presentes no cotidiano das famílias e as dúvidas crescem a cada dia.

Todos os aspectos envolvidos na complexidade existente na fibrose cística fazem com que a mãe passe por uma série de dificuldades, sejam elas financeiras, psicológicas e/ou sociais, e que são marcadas pelo sofrimento advindo do próprio diagnóstico, número reduzido de locais especializados para o tratamento, preconceito das pessoas, frequentes hospitalizações, entre outras. Entender um pouco mais sobre a realidade desses familiares (mãe) possibilita ao profissional de saúde criar abordagens mais precisas e que auxiliem essas mulheres, contribuindo para um cuidado pleno e eficaz (REIS AT, et al., 2016).

O cotidiano de um portador de FC muitas vezes é determinado por hospitalizações, cirurgias, limitações físicas, dependência de tecnologia e o transplante pulmonar. Assim, percebe-se que pacientes e familiares vivenciam a morte de forma mais presente ou até mesmo cotidiana, dessa forma, manter os padrões de vivência familiar se torna cada vez mais difícil e, de tal forma, acaba-se observando até o distanciamento familiar (AFONSO SBC e MITRE RMA, 2013).

As mudanças psicológicas dos portadores de FC assim como nas mães podem incluir depressão, tristeza, apatia, medo de iniciar novos relacionamentos românticos e incerteza em relação ao futuro. Portanto, é possível enumerar diversos sentimentos envolvidos, principalmente, aos que convivem com as dificuldades habituais associadas à adolescência ou aos cuidados de família (AGUIAR KCA, et al., 2016).

As necessidades subjetivas, baseadas no novo conceito de saúde incluíram o desejo de igualdade e aceitação. A busca de uma identidade pessoal ocorre no início da adolescência e pode ser bloqueado pela presença de doenças crônicas. Para ter sucesso, os adolescentes e crianças precisam sentir-se aceitos como iguais a seus pares, muitas vezes, as mães são ferramentas essenciais para a melhoria da autoestima dos portadores de síndrome. Este sentimento de ter uma identidade pode ter duas formas: a primeira é ver a si mesmo como os outros e de maneira contínua no tempo e no espaço e reconhecer-se como parte de um grupo (AGUIAR KCA et al., 2016).

Segundo o estudo de Afonso SBC e Mitre RMA (2013), em vários exemplos, o homem, quando o casal se encontrava separado, não se fazia presente de nenhuma forma. Já nos casos onde os casais encontravam-se juntos os homens não mantinham relação próxima a mãe e a criança. Dessa forma, é possível observar que inúmeros são os determinantes que podem influenciar negativamente o "andar" da doença. A presença da perspectiva da morte prematura da criança faz com que a família compartilhe sentimentos de medo, angústia e ansiedade, pois se trata de um período incerto e marcado por variações no estado de saúde dos portadores da doença.

A família ainda é considerada uma peça fundamental na melhoria de saúde de qualquer indivíduo e é a partir desse referencial que são formados os parâmetros de aceitabilidade da patologia, assim como seu tratamento, percebe-se é necessário um visão holística para efetivar um cuidado exemplar (COSTA ASM, et al., 2010).

Os enfermeiros incentivam as mães na escolha das melhores formas de vivência e ajuda aos filhos portadores da fibrose cística, auxiliando-as na escolha correta de técnicas e até na forma de se expressar e ouvir seus filhos, principalmente, quando se discuti a gravidade da doença. O contato dos enfermeiros com a fibrose cística é uma variável diversificada que apresenta vários sentimentos no seu crescimento e desenvolvimento, e está relacionado às limitações da condição crônica que acompanham os adolescentes em sua vida diária (REIS AT, et al., 2016). 
A assistência à criança com fibrose cística hospitalizada precisa ir além de cumprir um protocolo técnico de atendimento à doença e avaliar o que nem sempre é tão fácil de observar. Essa idéia converge com a atual visão de se considerar a família também como um dos focos de cuidado, onde esta é considerada indispensável ao cuidado integral do paciente e essencial na jornada do cuidado. Em meio ao sofrimento, as mães sentem extrema necessidade de apoio emocional e, muitas vezes, o encontram nos próprios profissionais de saúde (REIS AT, et al., 2016).

O conhecimento científico é essencial para a oferta de um cuidar holístico e humanizado que possa identificar todas as variedades de sinais e sintomas dos portadores de Fibrose Cística. A equipe de saúde, geralmente, direciona suas abordagens para o corpo biológico do indivíduo com doença crônica, enfatizando os aspectos técnicos do cuidar. Muitas vezes se esquece das necessidades individuais dessas pessoas e as de seus familiares, principalmente pela representatividade das doenças crônicas estabelecidas (REISINHO MCMSRO e GOMES BP, 2016).

\section{CONSIDERAÇÕES FINAIS}

A revisão da literatura revelou as características do processo de Enfermagem aos jovens portadores de FC, assim como os sentimentos envolvidos com a presença dos pais/família, considerando que o planejamento da assistência de Enfermagem deve priorizar não uma doença, mas o contexto subjetivo e familiar dos envolvidos dentro desse processo. Torna-se necessária a busca de manobras que possam aprimorar o cuidado do enfermeiro (a) a crianças e aos adolescentes com fibrose cística, aprendendo assim sobre o dinamismo do cuidar.

\section{REFERÊNCIAS}

1. AFONSO SBC, MITRE RMA. Notícias difíceis: sentidos atribuídos por familiares de crianças com fibrose cística. Ciênc. saúde coletiva, Rio de Janeiro, 2013; 18(9): 2605-2613.

2. AGUIAR KCA, et al. Viver com fibrose cística: a visão pessoal do adolescente brasileiro. Psicologia em Estudo, 2016; 21(2): 211-222.

3. CABIZUCA M, et al. Os pacientes invisíveis: transtorno de estresse pós-traumático em pais de pacientes com fibrose cística. Rev. psiquiatr. clín., São Paulo, 2010; 37(1): 6-11.

4. COSTA ASM, et al. Vivências de familiares de crianças e adolescentes com fibrose cística. Rev. bras. crescimento desenvolv. hum., São Paulo, 2010; 20(2): 217-227.

5. REIS AT, et al. Vivências de mães que acompanham filhos com fibrose cística no hospital: subsídios para a enfermagem. Rev. Ciênc. Méd., Campinas, 2016; 25(2): 49-56.

6. REISINHO MCMSRO, GOMES BP. Intervenções de enfermagem no monitoramento de adolescentes com fibrose cística: uma revisão da literatura. Rev. Latino-Am. Enfermagem, Ribeirão Preto, 2016; 24.

7. SCHRAM C. Atypical cystic fibrosis: Identification in the primary care setting. Can Fam Physician, 2012; 58: v. 58: 1341-5.

8. SILVA A, et al. Histórico, avanços no diagnóstico e tratamento da fibrose cística. III Simpósio de assistência farmacêutica, 2015.

9. TAVARES KO, et al. O que é ser mãe de uma criança com fibrose cística. Rev. Gaúcha Enferm., Porto Alegre, 2010; 31(4): 723-729. 